\title{
EVALUATION OF IMPROVEMENT OPPORTUNITIES FOR THE COLLABORATION OF DESIGN AND SIMULATION - AN INDUSTRIAL MULTI-METHOD STUDY
}

\author{
Schweigert-Recksiek, Sebastian; \\ Hagenow, Niklas; \\ Lindemann, Udo \\ Technical University of Munich
}

\begin{abstract}
As mechanical simulations play an increasingly role in engineering projects, an appropriate integration of simulations into design-oriented product development processes is essential for efficient collaboration. To identify and overcome barriers between design and simulation departments, the BRIDGES approach was elaborated for barrier reduction in design engineering and simulation. This paper shows the industrial evaluation of the approach using a multi-method study of an online survey and focus group workshops. The experts' assessments were statistically analysed to build a connection matrix of barriers and recommendations. 59 participants from multiple industries with practical experience in the field contributed to the online survey, while 24 experts could be acquired for the focus group workshops. As a result of the workshops, both the data-based and the workshop-based part of the BRIDGES approach were assessed as beneficial to raise the efficiency of collaboration and practically applicable. This provides an empirically secured connection of barriers and suitable recommendations, allowing companies to identify and overcome collaboration barriers between design and simulation.
\end{abstract}

Keywords: Communication, Complexity, Simulation, Expert Workshops, Statistical Analysis

\section{Contact:}

Schweigert-Recksiek, Sebastian

Technical University of Munich

Product Development and Lightweight Design

Germany

schweigert.recksiek@tum.de

Cite this article: Schweigert-Recksiek, S., Hagenow, N., Lindemann, U. (2021) 'Evaluation of Improvement

Opportunities for the Collaboration of Design and Simulation - An Industrial Multi-Method Study', in Proceedings of the International Conference on Engineering Design (ICED21), Gothenburg, Sweden, 16-20 August 2021. DOI:10.1017/ pds.2021.142 


\section{INTRODUCTION}

Mechanical simulations of technical products have been gaining increasing importance in today's product development process (PDP). Both, the number and the complexity of simulations have drastically increased and are expected to continue that way (Norris, 2017) . To integrate simulations into the mostly design-oriented product development process, Deubzer et al. (2005) identified four core dimensions for a holistic CAD-CAE integration: product, people, data, and tools. The latter three aspects can be regarded as forming the fifth dimension, process, which should be treated as a separate dimension according to Kreimeyer et al. (2005). To enhance the integration of simulations into the PDP with special emphasis on the dimensions of people and processes, the so called BRIDGES appraoch (Barrier Reduction in DesiGn Engineering and Simulation) has been developed (cf. Figure 1), facilitating the identification of collaboration barriers (e.g. Difficulties in concurrent engineering), and suggesting appropriate recommendations for improvement measures (e.g. Effort and good information transmission in the initial phases). The approach includes the options of a data-based, as well as a workshop-based application (cf. Schweigert-Recksiek and Lindemann (2020)).
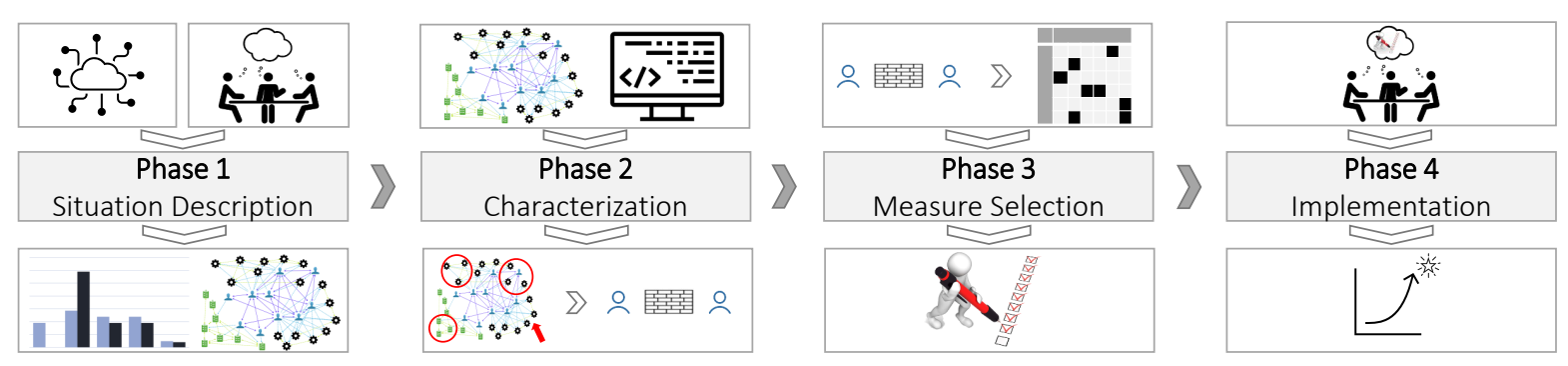

Figure 1. BRIDGES approach (adapted from Schweigert-Recksiek and Lindemann 2020)

The BRIDGES approach, as shown in Figure 1, consists of four phases: situation description, characterization, measure selection, and implementation. The upper row of Figure 1 shows the inputs for each phase, while the outputs are depicted below the phases. In the situation description of phase 1, workshops, checklists, and data analysis are used to understand the current state of collaboration of the company in question. The current state is then analyzed in the characterization of phase 2 to identify possible barriers in the collaboration of design and simulation. The measure selection of Phase $\mathbf{3}$ is the crucial part for the improvement of the collaboration of design and simulation. The main contribution of the approach is the mapping of barriers and suitable recommendations to overcome the barriers. Finally, this is the input for repeated workshops in the implementation of phase 4, where the final measures for implementation are selected with the aim of improving the overall efficiency of the collaboration of design and simulation. This paper describes the process and the result of a multi-method study to evaluate the BRIDGES approach with respect to practical applicability as well as usefulness to increase the efficiency of collaboration of design and simulation.

\section{STATE OF THE ART AND RESEARCH}

\subsection{Collaboration of Design and Simulation}

As stated in the introduction, previous work on the collaboration of design and simulation has been conducted by Deubzer et al. (2005) and Herfeld et al. (2006), who analyzed communication between design and simulation departments. They state that different objectives of the two disciplines are the main obstacle in their collaboration. Most other literature sources in this area focus on the tools dimension of integration. Schönwald et al. (2019), for example, revealed in an empirical study that simulation data management tools are very beneficial, and more widely used than, for example, test data management. Eriksson et al. (2014) and Petersson et al. (2013) conducted an interview study on the current state of integration of simulation into the product development process. They focused on technical aspects (data and tools in the dimensions of Deubzer et al. (2005)), and emphasize for example that CAD-integrated FEM systems are used systematically in design departments of a high number of companies, and that these simulations are performed efficiently. Little research has been conducted to improve the collaboration of design and simulation in the dimensions of people and processes. 


\subsection{Structural Collaboration Analysis}

The data-based part of the BRIDGES approach (mainly phase 2 in Figure 1) uses structural collaboration analysis to asses the collaboration of design and simulation. With similar methods, Piccolo et al. (2018) build multiple domain matrices of structural engineering project data to correlate properties of tasks and execution time. They make use of a big dataset of e-mail data to enable an analysis of the underlying engineering project that lasted several years. Several further studies of structural collaboration analysis are described in Eppinger and Browning (2012), as well as by Snider et al. (2017). However, none of them specifically focuses on the collaboration of design and simulation.

\subsection{Preliminary Work}

As this paper describes the final evaluation of a research approach originating in a multi-year $\mathrm{PhD}$ project, the BRIDGES approach cannot be described in detail here. Table 1 lists previous publications with more detailed descriptions of the approach.

Table 1. Previous publications on the BRIDGES approach

\begin{tabular}{|c|c|}
\hline Publication & Content \\
\hline Schweigert et al. (2017a) & Initial publication of the overall approach \\
\hline Schweigert et al. (2017b) & $\begin{array}{l}\text { Description of the first online survey on the current state of collaboration with } 79 \\
\text { participants from industry }\end{array}$ \\
\hline $\begin{array}{l}\text { Schweigert-Recksiek and } \\
\text { Lindemann (2018) }\end{array}$ & $\begin{array}{l}\text { Identification of the barriers resulting from an industrial interview study with } 16 \\
\text { participants }\end{array}$ \\
\hline Schweigert-Recksiek et al. (2019) & $\begin{array}{l}\text { Statistical analysis of the data from the survey described in (Schweigert et al., 2017b), } \\
\text { resulting in the initial connection matrix of barriers and recommendations }\end{array}$ \\
\hline Schweigert-Recksiek et al. (2020) & Initial evaluation of the data-based approach in an agile medical engineering project \\
\hline $\begin{array}{l}\text { Schweigert-Recksiek and } \\
\text { Lindemann (2020) }\end{array}$ & $\begin{array}{l}\text { Elaboration of the approach and list of recommendations as well as workshop-based } \\
\text { case study with an industry partner }\end{array}$ \\
\hline
\end{tabular}

An initial connection matrix, mapping recommendations to barriers (cf. Schweigert-Recksiek et al. (2019)) laid the foundation for the evaluation study of this paper. Based on the results of the evaluation study, it was substituted with a final connection matrix (cf. Figure 8 in subsection 4.2 below).

\section{METHODOLOGY - EVALUATION CONCEPT}

\subsection{Research Goals}

The overall goal of the presented study was the assessment of the elaborated BRIDGES approach. As the data-based approach has already been evaluated (cf. Schweigert-Recksiek et al. 2020), the presented evaluation study focuses on the workshop-based approach. Following the Design Research Methodology (DRM) by Blessing and Chakrabarti (2009), the approach is evaluated with respect to its applicability, and its overall usefulness for the support of the design process. For this purpose, the following research questions were defined:

RQ1: To what extent are the proposed recommendations suitable to reduce the associated barriers in the collaboration of design and simulation?

RQ2: To what extent are the proposed recommendations suitable to improve the overall efficiency of collaboration of design and simulation?

RQ3: To what extent is the developed approach applicable in a practical environment?

\subsection{Study Design}

The evaluation study (cf. Figure 2) was performed with a convenience sample of experts, mainly from the German industry. The sampling criteria were expert-level knowledge in the field of engineering design or simulation, as well as professional experience in technical product development. The study encompassed two major phases, simulating the application of the workshop-based BRIDGES approach:

Phase 1: An online-survey

Phase 2: A series of focus group workshops 
The main purpose of the online-survey was to gather quantitative data on the extent to which the 20 barriers included in the BRIDGES approach are present in the experts' companies, and to which extent the 16 recommendations are already in place. Additionally, the participants were asked to rate their perceived level of efficiency in the collaboration of design and simulation in their company. In a practical application of the workshop-based BRIDGES approach, this data would be used to identify relevant barriers and assess the as-is efficiency of collaboration in the observed company. Within the scope of the presented evaluation study, the collected data was used to validate the associations between the barriers and the recommendations from the initial connection matrix (cf. subsection 4.2).

All survey participants $(\mathrm{N}=59)$ from an initially contacted convenience sample $(\mathrm{N}=99)$ were asked to further participate in one of six subsequent focus group workshops. A subset of 25 participants could be acquired for this second phase of the study. The main goal of the workshop series was to let the experts discuss and rate the suitability, practical applicability, and usefulness of recommendations, as well as the practical applicability and usefulness of the overall BRIDGES approach.

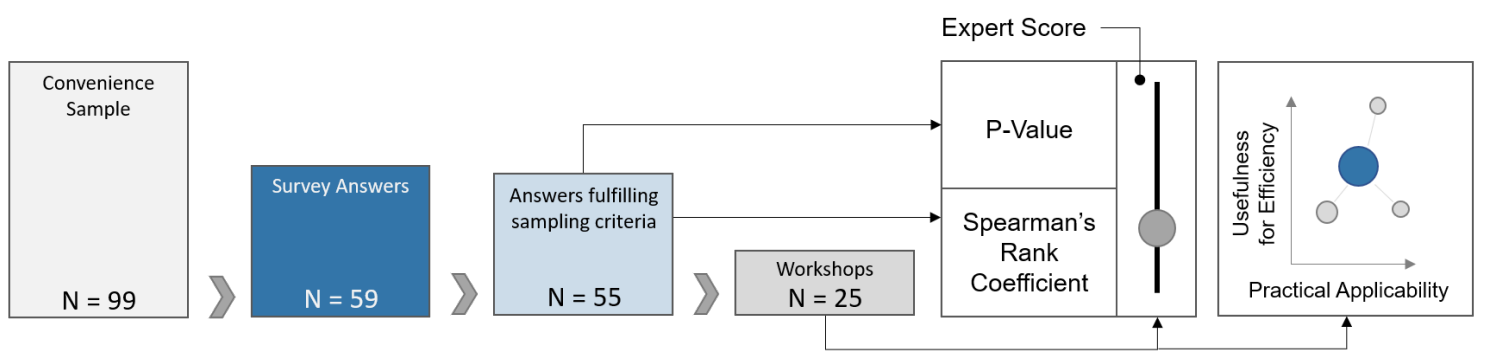

Figure 2. Study design

The results from this multi-method study provide both, a statistical view (p-value and Spearman's rank coefficient in Figure 2), as well as an expert view (expert score, practical applicability and usefulness for efficiency in Figure 2) on the applicability and the usefulness of the BRIDGES approach. These values are used to derive a final connection matrix of barriers and recommendations as illustrated in Figure 8.

\subsection{Online Survey}

The online-questionnaire designed for the survey was composed of four sections (cf. Figure 3): Section 1 contains questions to characterize the participants and to verify the fulfillment of the defined sampling criteria. Section 2 asks for the participants' perception of the existence of each of the 20 barriers in their companies. In section 3, the participants rate the level to which their companies already work as proposed by each of the 16 recommendations. The fourth section asks for the perceived level of efficiency in the collaboration of design and simulation.

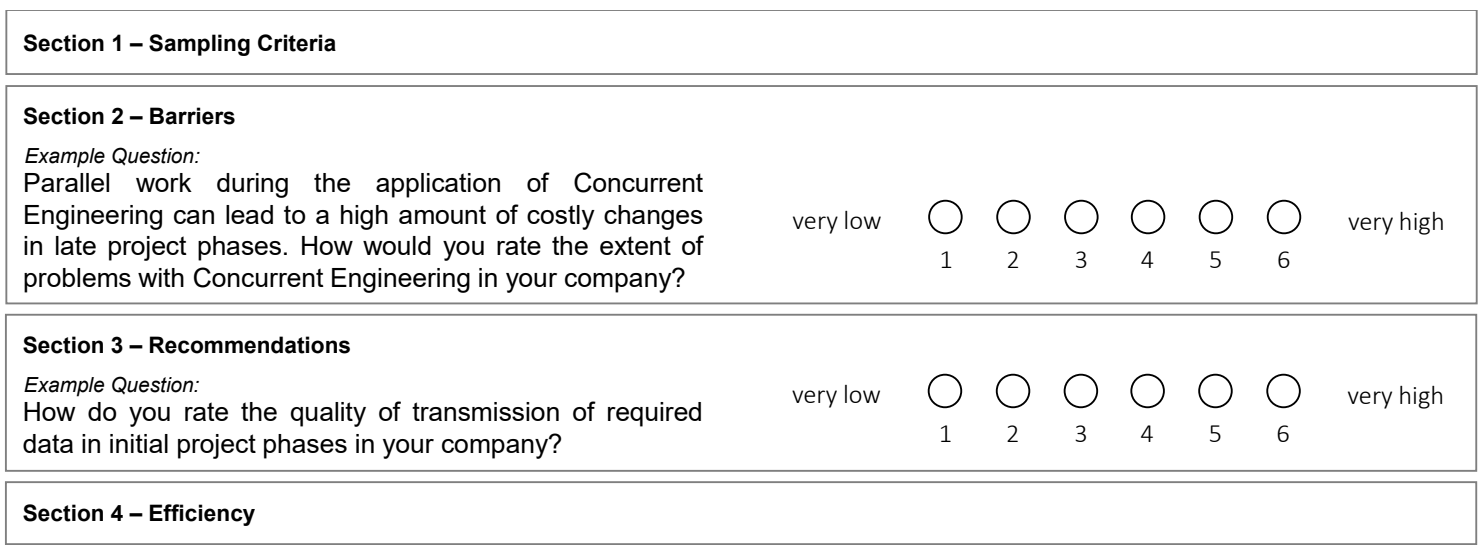

Figure 3. Survey structure

\subsection{Focus Group Workshops}

In order to simulate the application of the workshop-based part of the BRIDGES approach, each of the workshop participants had to be matched with other participants who share as many barriers as possible. 
This is essential as shared barriers enable discussions on a common ground so that the highest possible amount of expertise can be reached (Fern, 1982). For this purpose, a genetic algorithm was used to find a suitable combination of participants for a first series of four workshops, according to their availability and extent of barriers as rated in the questionnaire. Each of these four workshops covered four different barriers and the corresponding recommendations based on the initial connection matrix. The remaining four barriers were covered in two additional workshops with participants who previously could not be matched due to limited availability. As exceptions to this procedure, two workshop participants had not completed the survey upfront and were matched based on availability only. Figure 4 shows the structure of the six workshops.
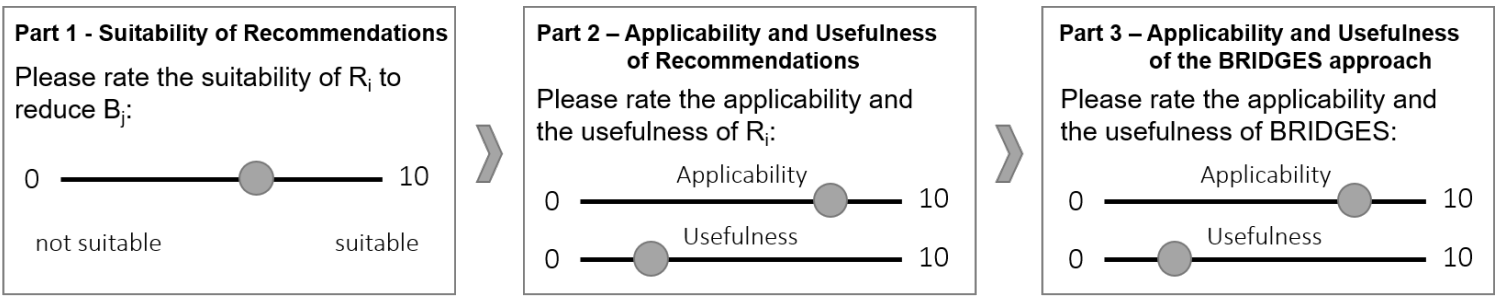

Figure 4. Workshop sequence

After an introduction to both, the data-based and the workshop-based part of the BRIDGES approach, the participants first assessed the suitability of recommendations to reduce the corresponding barriers. Further, in the second part, the recommendations were rated with respect to their practical applicability (costs, obstacles, etc.) and usefulness to improve the overall efficiency of collaboration. Conclusively, the participants also rated the data-based and the workshop-based BRIDGES approaches overall, with respect to their practical applicability and usefulness to improve the efficiency of collaboration of design and simulation. All ratings were given anonymously with real-time visualization of the contributions by using an interactive presentation tool. After each rating, the results were discussed by the experts in a focus-group-like manner (cf. Hennink (2014)). This finally resulted in applicability/usefulness portfolios of the recommendations as well as the overall approach that could directly be exported from the tool as well as usefulness ratings of the recommendations that were compared to the online-survey data manually.

\subsection{Statistical Analysis}

It is broadly argued, under which circumstances Likert-type scales, as used in the online-questionnaire, as well as the workshops, can deliver interval data rather than ordinal data. Since only the endpoints of the scales were labeled, it was assumed that the answer options are psychologically perceived as equally spaced and therefore the data can be treated as interval data as suggested by Hamby and Levine (2016). All ratings given by the experts during the workshop series on end-point-labeled Likert-type scales were therefore treated as interval data, allowing the calculation of mean values.

However, since not all answers given for the 20 barriers and 16 recommendations can be assumed to come from a normally distributed sample (e.g. for B09 the D'Agostino $\mathrm{K}^{2}$-Test yields: $K^{2}=6.87$, pvalue $=0.03$ ), a non-parametric approach was chosen to determine correlations between barriers and recommendations from the survey answers. For this purpose, the Spearman's rank coefficient was calculated along with the p-value of each pair of barrier and recommendation. Additionally, the same method was used to validate that the discovered barriers are negatively correlated with the efficiency of collaboration, and that the recommendations are overall positively correlated with a higher level of the efficiency in the collaboration of design and simulation.

\section{FINDINGS}

\subsection{Sample Profile and Extent of Barriers and Recommendations}

The study comprised experts from twelve different industries, defined in accordance with the Global Industry Classification Standard (GICS, www.msci.com/gics) (cf. Figure 5a). Each participant in the study gave a self-assessment of their level of expertise in the fields of engineering design and simulation as part of the online questionnaire. Included in the results were only those with a self-rating of at least 
three out of six points on the answer scale in either of the two fields. Four of 59 survey answers had to be excluded, as the self-assessment of the level of expertise was insufficient to meet the defined sampling criteria. The majority of participants are interdisciplinary experts with sufficient expertise in both, design and simulation (cf. Figure 5b).
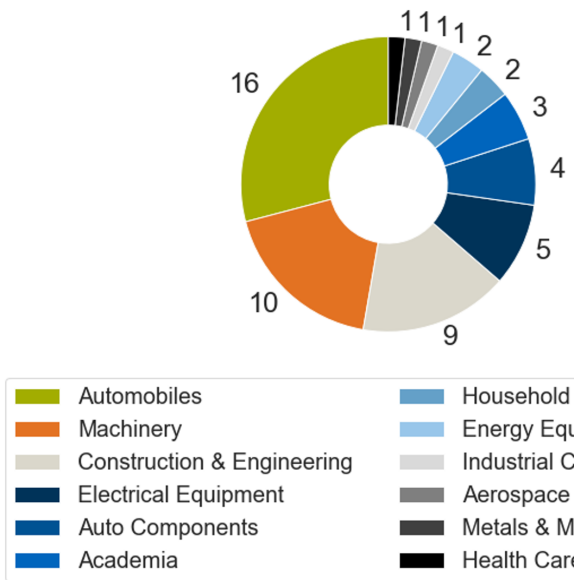

Household Durables
Energy Equipment \& Services
Industrial Conglomerates
Aerospace \& Defense
Metals \& Mining
Health Care Technology

(a) Industries ( $N=55)$
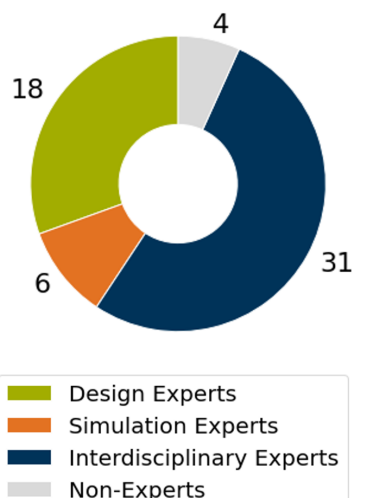

(b) Expertise ( $N=59)$

Figure 5. Sample profile

Figure 6 shows the participants' answers for the extent of the 20 barriers (Figure 6a), and the level to which their companies already work as proposed by each of the 16 recommendations (Figure 6b). The answers were consistently given on a scale from 1 (= very low) to 6 (= very high). The results show, for example, high extents of B20 (inefficient use of CAD-integrated FEM systems), while most participants feel like R12 (utilizing knowledge from experienced designers) is already well applied.

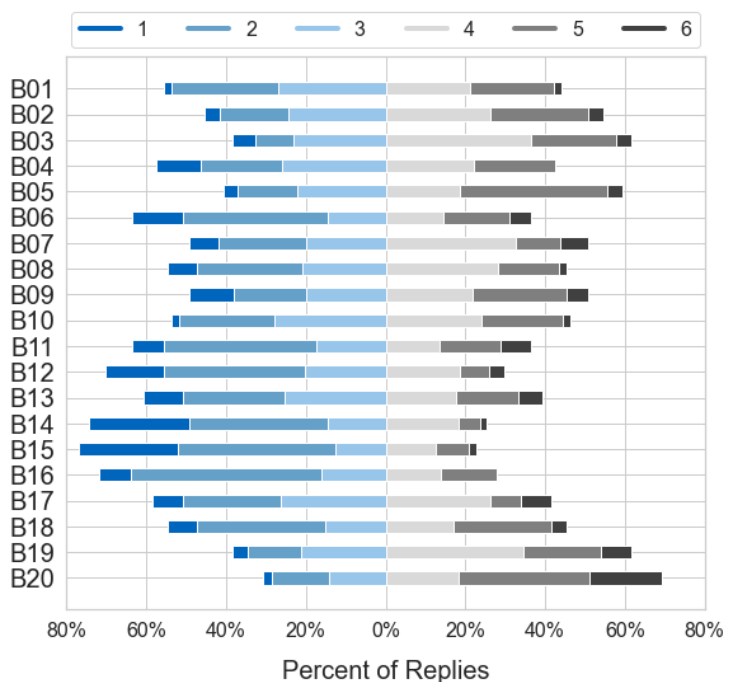

(a) Barriers

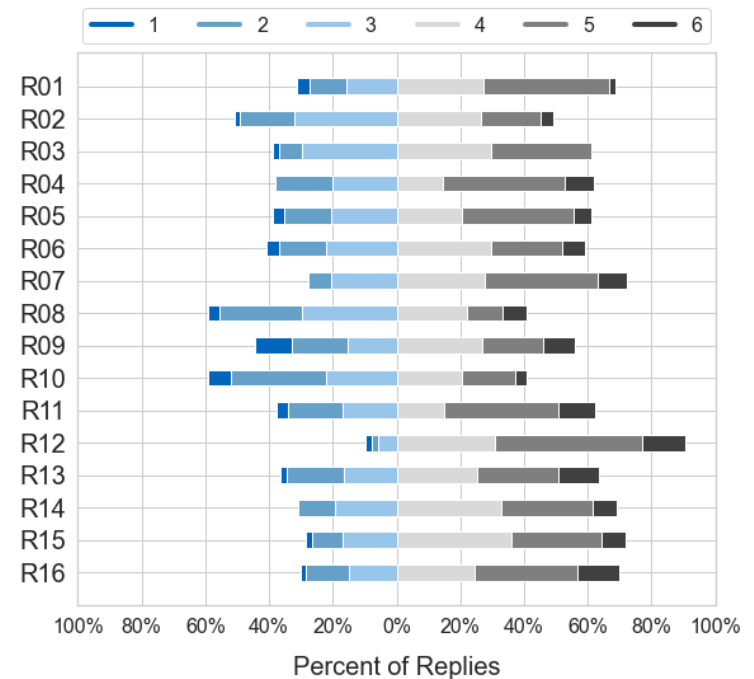

(b) Recommendations

Figure 6. Survey answers for the extent of barriers and recommendations

\subsection{Connection between Barriers and Recommendations}

In order to validate the connections between barriers and recommendations from the initial connection matrix, three criteria were defined (cf. Figure 7). A connection is regarded as validated if the statistical analysis shows a Spearman's rank coefficient $\leq-0.25$ and a p-value $<0.05$, as well as an expert score of $\geq 5$ out of 10 , if the connection was assessed in the workshop series.

The resulting matrix (cf. Figure 8) shows the validated connections (highlighted) between barriers and recommendations. The upper left value in each cell shows the p-value (rounded up to two decimal points) of the connection, the lower left value shows Spearman's rank coefficient (rounded up to two decimal points), and the right scale the expert score on a scale from zero (bottom position) to ten (top 


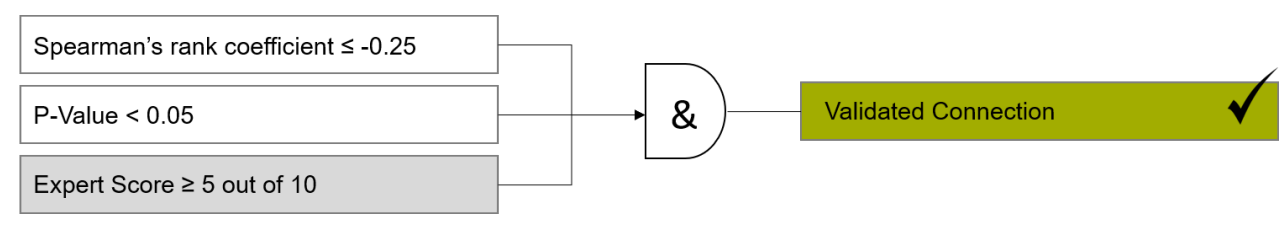

Figure 7. Validation criteria

position). Since only the proposed connections from the initial matrix (cf. subsection 2.3, taken from Schweigert-Recksiek et al. (2019)) were evaluated in the workshop series, newly discovered correlations that were not assessed by the experts but fulfill the first and the second criterion are included in the resulting matrix as well.

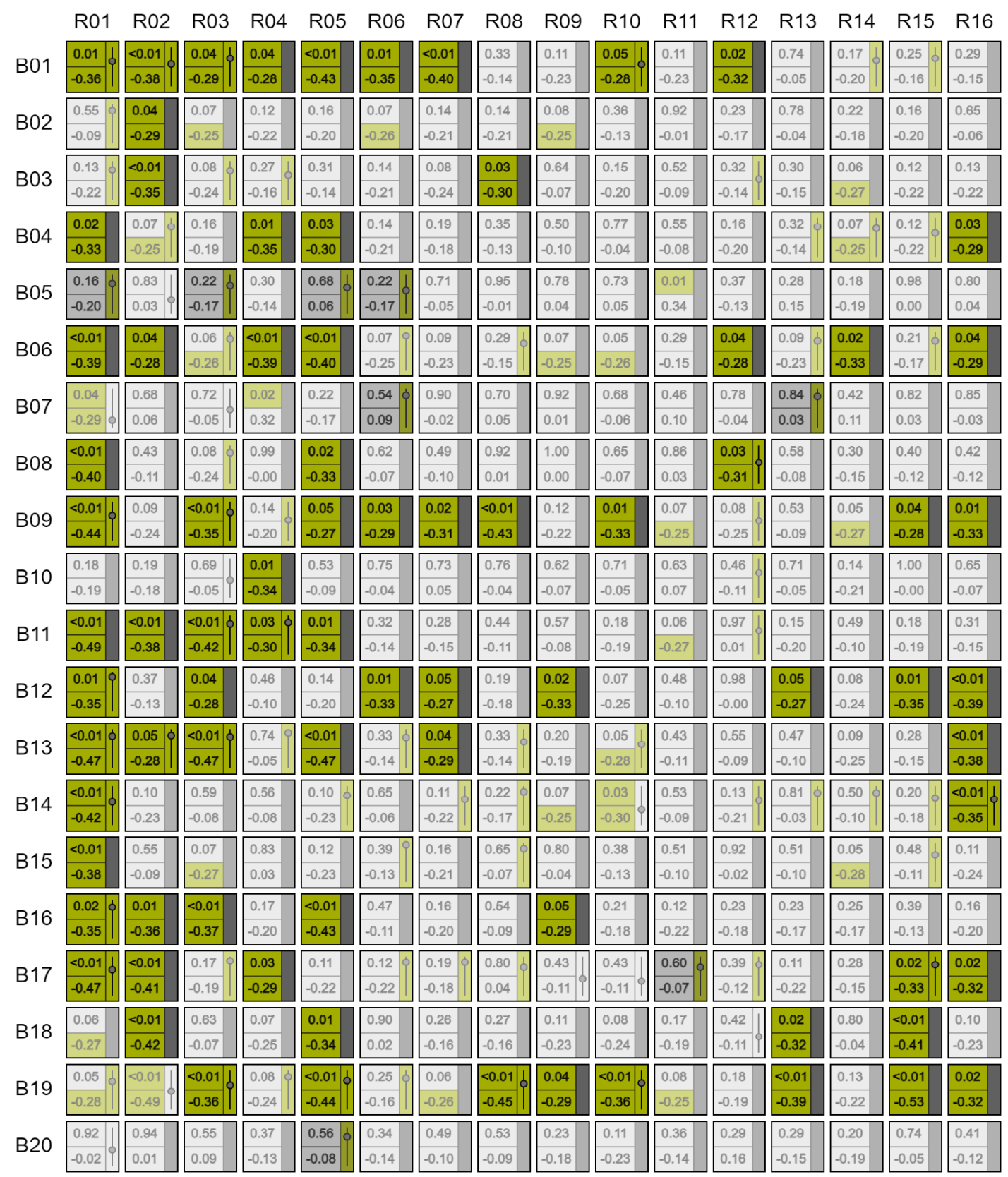

Figure 8. Detailed connection matrix of barriers and recommendations

As result, 22 out of 83 connections from the initial matrix were validated. 61 out of 83 connections could not be confirmed. 58 connections were newly discovered. For three barriers (B05, B07 and B20) and for recommendation R11, no connection fulfilling the defined criteria was found. However, the expert score suggest the suitability of a least one recommendation for each of these barriers, as well as the connection with one barrier (B17) for R11 (highlighted in Figure 8). Figure 9 summarizes the resulting 


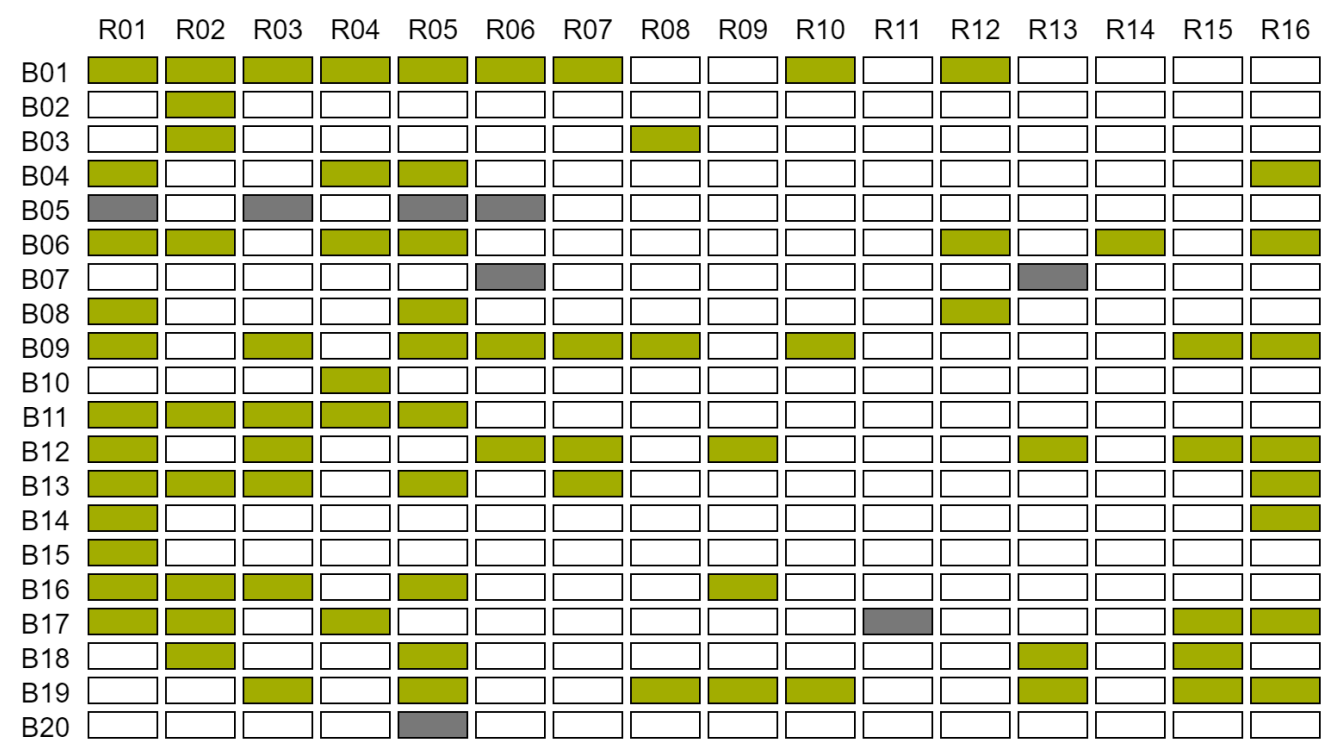

Figure 9. Connection matrix of barriers and recommendations

connections in a final connection matrix, including those fulfilling the criteria from Figure 7 (green), and the improvised connections for B05, B07, B20 and R11 (grey).

\subsection{Evaluation of Recommendations}

Besides the suitability to reduce a particular barrier, the experts also positioned each recommendation in a two-dimensional grid to rate the recommendations' general usefulness to improve the efficiency of collaboration, and the practical applicability with respect to costs and potential obstacles in the implementation. Figure 10a shows the mean positioning of each recommendation, while Figure 10b shows the mean and the individual positioning for R06 (increase the interaction of the disciplines and support the exchange of questions, ideas, and values) as an example.

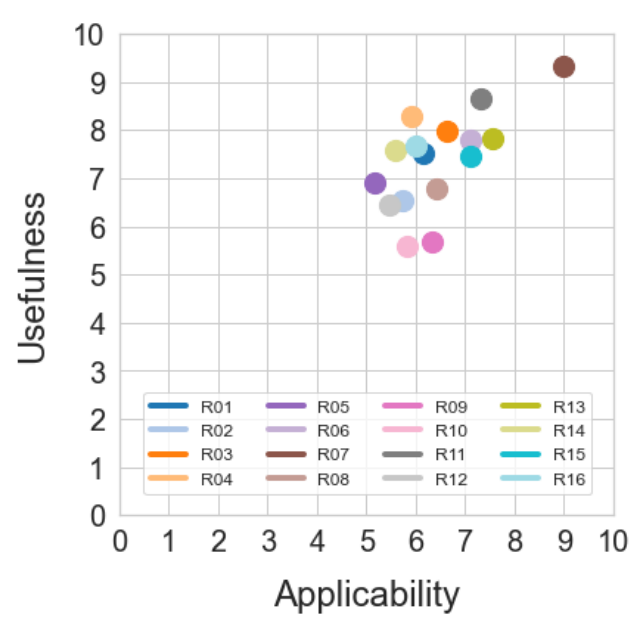

(a) Mean scores for all 16 recommendations

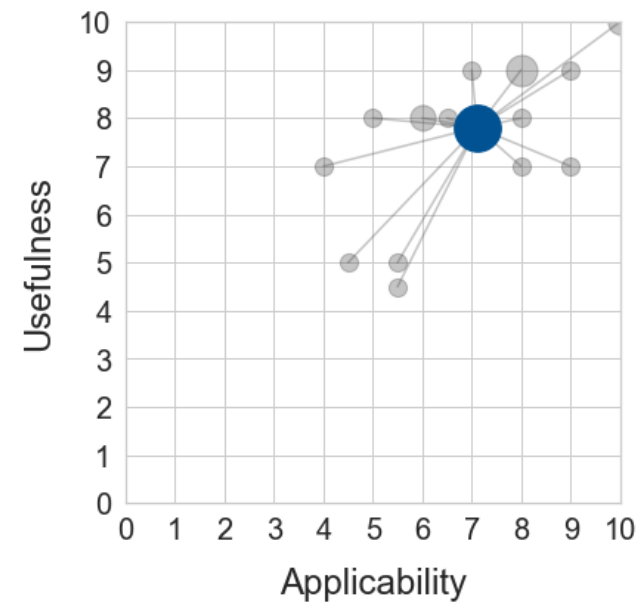

(b) Mean and individual scores for R06

Figure 10. Applicability and usefulness of recommendations

As Figure 10a shows, all of the 16 recommendations are positioned in the upper-right quadrant of the grid, implying high levels of usefulness and practical applicability. R07 (pro-activity in dealing with conflicts and solution-oriented communication) has been rated particularly high in both dimensions.

\subsection{Evaluation of the overall BRIDGES Approach}

As for the recommendations, the general usefulness to improve the efficiency of collaboration and the practical applicability with respect to costs and potential obstacles in the implementation of the overall BRIDES approach were rated by the experts. The data-based approach was rated based on a theoretical 
introduction given by the researchers only, while the workshop-based approach was assessed based on the experts' experiences during the participation in the evaluation study, which simulated the workshopbased application. Figure 11 shows the positioning of both approaches in the two-dimensional grid.

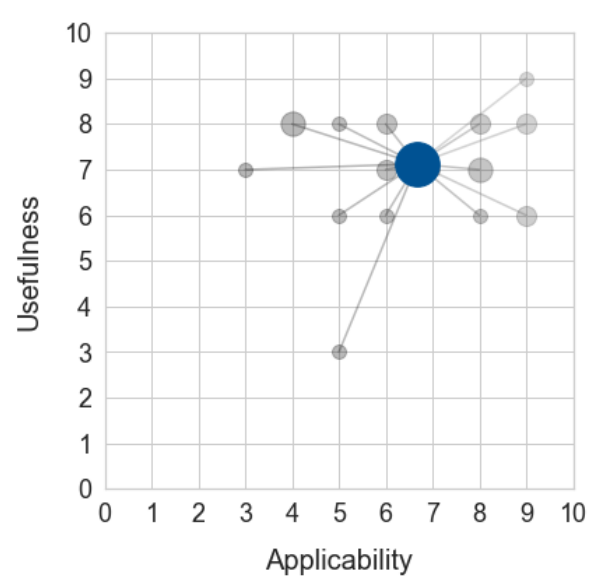

(a) Workshop-based approach

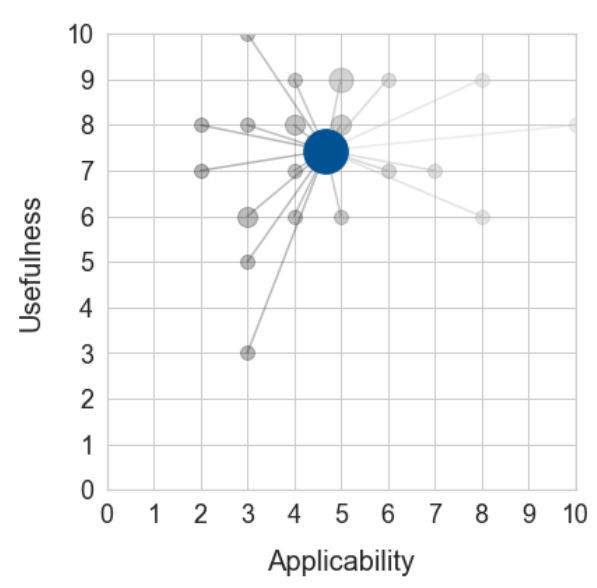

(b) Data-based approach

Figure 11. Applicability and usefulness of the BRIDGES approach

While the data-based approach was rated slightly higher with respect to usefulness, the workshop-based approach received higher scores in the applicability dimension. The experts expressed potential legal issues with the collection of personal data as a major concern for a data-based application, mitigating the practical applicability of the approach. The highest benefit of the workshop-based approach was seen in the fact that "the right people discuss the right topics" in a proper setting.

\section{CONCLUSION}

\subsection{Discussion}

The relatively small number of participants $(\mathrm{N}=59)$ limits the general validity of the results. Furthermore, a convenience sample was chosen based on the researchers' professional network due to the limited availability of experts. This practice could potentially promote a social desirability bias. However, as all of the participants included can be regarded as experts in their field, the study is still grounded on a rather large sample compared to other studies in the field of engineering design. As a result, the findings of the evaluation study prove the capability of the BRIDGES approach to enhance the collaboration of design and simulation with regard to all three dimensions of the evaluation. Thus, it is possible to recommend suitable improvement measures for all typical barriers in the collaboration of design and simulation, leading to a high degree of applicability and usefulness as proven in a study with multiple experts from a great range of industrial backgrounds.

\subsection{Outlook}

The statistical methods that were used to create the connection matrix between barriers and recommendations took into account the connection between two variables - one barrier and recommendation each. It has therefore not yet been investigated if the combination of multiple recommendations might have a positive effect on the reduction of a barrier, while the sole application of single measures does not have a statistically relevant effect on the barrier in question.

Now that the BRIDGES approach was extensively evaluated in the presented multi-method study, as well as in an initial academic application (cf. Schweigert-Recksiek et al. (2020)), what is missing is an industrial application of the approach on a large dataset.

\section{ACKNOWLEDGMENTS}

The authors are very thankful to all the participants in both, the survey and especially the workshops. The number and motivation of participants exceeded the authors' expectations. 


\section{REFERENCES}

Blessing, L.T. and Chakrabarti, A. (2009), A design research methodology, Heurista, 10.1007/978-1-84882-587-1.

Deubzer, F., Herfeld, U., Kreimeyer, M. and Lindemann, U. (2005), “A structured holistic approach for the integration of cad and cae environments", ProSTEP iViP Science Days 2005.

Eppinger, S.D. and Browning, T.R. (2012), Design structure matrix methods and applications, MIT press, 10.7551/mitpress/8896.001.0001.

Eriksson, M., Petersson, H., Bjärnemo, R. and Motte, D. (2014), "Interaction between computer-based design analysis activities and the engineering design process-an industrial survey”, in: D. Marjanović, M. Štorga, N. Pavković and N. Bojčetić (Editors), Proceedings of the DESIGN 2014, 13th International Design Conference, Dubrovnik, Croatia, Vol. 3.

Fern, E.F. (1982), "The Use of Focus Groups for Idea Generation: The Effects of Group Size, Acquaintanceship, and Moderator on Response Quantity and Quality”, Journal of Marketing Research, Vol. 19 No. 1, pp. $1-13,10.2307 / 3151525$.

Hamby, T. and Levine, D.S. (2016), "Response-Scale Formats and Psychological Distances Between Categories", Applied psychological measurement, Vol. 40 No. 1, pp. 73-75, 10.1177/0146621615597961.

Hennink, M.M. (2014), Focus Group Discussions, Understanding qualitative research, Oxford University Press, Oxford.

Herfeld, U., Kreimeyer, M., Deubzer, F., Frank, T., Lindemann, U. and Knaust, U. (2006), "Verknüpfung von komponenten und funktionen zur integration von konstruktion und simulation in der karosserieentwicklung", VDI-Berichte, No. 1967, pp. 259-276.

Kreimeyer, M., Deubzer, F., Herfeld, U. and Lindemann, U. (2005), “A survey on efficient collaboration of design and simulation in product development", in: Proceedings 23rd CADFEM Users' Meeting.

Norris, M. (2017), “How to succeed @ spdm”, in: International SPDM Conference, Stockholm.

Petersson, H., Eriksson, M. and Bjärnemo, R. (2013), "Integration of computer aided design analysis into the engineering design process for use by engineering designers", in: ASME International Mechanical Engineering Congress and Exposition, 10.1115/imece2013-62130.

Piccolo, S., Trauer, J., Wilberg, J. and Maier, A. (2018), "Understanding task execution time in relation to the multilayer project structure: Empirical evidence", in: C. Leardi, T. Browning, S.D. Eppinger and L. Becerril (Editors), Proceedings of the 20th International Dependency and Structure Modeling (DSM) Conference, Trieste (Italy), pp. 129-138, 10.21278/idc.2018.0283.

Schönwald, J.R., Forsteneichner, C., Vahrenhorst, D. and Paetzold, K. (2019), "Improvement of collaboration between testing and simulation departments on the example of a motorcycle manufacturer", in: Proceedings of the Design Society: International Conference on Engineering Design, Vol. 1, Cambridge university press, pp. 149-158, 10.1017/dsi.2019.18.

Schweigert, S., Luft, T., Wartzack, S. and Lindemann, U. (2017a), “Combination of matrix-based and graph-based modeling for product and organizational structures", in: K. Hölttä-Otto, T. Browning, S. Eppinger and L. Becerril (Editors), Proceedings of the 19th International DSM Conference.

Schweigert, S., Xia, M. and Lindemann, U. (2017b), "An empirical survey on efficiency improvement for the collaboration between design and simulation departments", in: A. Maier (Editor), Proceedings of the 21st International Conference on Engineering Design, Vol. Vol 2: Design Processes, Design Organisation and Management, pp. 337-346.

Schweigert-Recksiek, S., Idrissov, A., Bharadwaj, T. and Lindemann, U. (2020), “Graph-based collaboration analysis of an agile medical engineering project with structural metrics”, in: S.D. Eppinger, T. Browning and J. Trauer (Editors), Proceedings of the 22nd International DSM Conference (DSM 2020), MIT, Cambridge, Massachusetts, 13.-15.10.2020, The Design Society, p. 10, 10.35199/dsm2020.20.

Schweigert-Recksiek, S., Koch, C. and Lindemann, U. (2019), "Matrix-based multivariate analysis of survey data on potentials for the collaboration of design and simulation", in: Proceedings of the 21st International DSM Conference, Monterey, California, pp. 3-13, 10.35199/dsm2019.1.

Schweigert-Recksiek, S. and Lindemann, U. (2018), "Improvement opportunities for the collaboration of design and simulation departments-an interview study", in: Proceedings of INTERNATIONAL DESIGN CONFERENCE - DESIGN 2018, pp. 905-916, 10.21278/idc.2018.0393.

Schweigert-Recksiek, S. and Lindemann, U. (2020), "Choosing the right measures to improve collaboration collaboration between design and simulation departments", in: Proceedings of the INTERNATIONAL DESIGN CONFERENCE - DESIGN 2020, 10.1017/dsd.2020.57.

Snider, C., Škec, S., Gopsill, J.A. and Hicks, B.J. (2017), “The characterisation of engineering activity through email communication and content dynamics, for support of engineering project management", Design Science, Vol. 3, 10.1017/dsj.2017.16. 